\title{
Measurement-based Analysis of Dense Multipath Components in a Large Industrial Warehouse
}

\author{
B. Hanssens ${ }^{1}$, S. R. Kshetri ${ }^{1}$, E. Tanghe ${ }^{1}$, D. Plets ${ }^{1}$, J. Hoebeke $^{2}$, A. Karaağaç ${ }^{2}$, \\ J. Haxhibeqiri ${ }^{2}$, D. P. Gaillot ${ }^{3}$, M. Liénard ${ }^{3}$, C. Oestges ${ }^{4}$, L. Martens ${ }^{1}$, W. Joseph ${ }^{1}$ \\ ${ }^{1}$ INTEC-WAVES, Ghent University / imec, Ghent, Belgium. Brecht.Hanssens@UGent.be \\ ${ }^{2}$ INTEC-IDLab, Ghent University / imec, Ghent, Belgium. Jeroen.Hoebeke@UGent.be \\ ${ }^{3}$ IEMN/TELICE, University of Lille 1, Lille, France. Davy.Gaillot@Univ-Lille1.fr \\ ${ }^{4}$ ICTEAM, Université catholique de Louvain, Louvain-la-Neuve, Belgium. Claude.Oestges@UCLouvain.be
}

\begin{abstract}
This work presents a measurement-based polarimetric analysis of the specular- and dense multipath components (SMCs and DMC) in a large industrial warehouse. MIMO channel sounding measurements were carried out in a flower auction warehouse, and were processed with the RiMAX multipath estimation framework. In the analysis, we investigate the behavior of the SMCs and the DMC power as a function of both the polarization and the transmitter-receiver distance for 1507 distinct positions of transmitter and receiver. The cross-polar discrimination of the transmitted $\mathrm{V}$ and $\mathrm{H}$ polarizations were analyzed statistically for this environment, as well as the relative power ratio of the DMC, and the DMC reverberation time.
\end{abstract}

Index Terms-Channel Modeling, Multipath Estimation, RiMAX, Polarization, Dense Multipath Components, DMC

\section{INTRODUCTION}

Over the recent years, innovations in wireless communication technologies have increasingly gained popularity for industrial applications. The term "Industry 4.0" summarizes the current trend of automation and data exchange in manufacturing technologies, and contains a set of design principles to achieve this goal such as interoperability, information transparency, technical assistance and decentralized decisions. Technologies such as IEEE 802.11 (Wi-Fi), LoRa [1], and ZigBee [2] can offer intelligent wireless solutions for communication, tracking and localization in industrial warehouses. These environments benefit immensely from the recent improvements in automation, production and logistics, allowing for reduction in manual labor for operators, and better and faster execution of industrial processes.

The wireless communication network plays an important role in this process. If such a network has dynamic information about the location of products and their storage places, it can optimally utilize resources such as time and manpower, pushing the efficiency of these warehouses even further. In order to stay competitive, industrial warehouses are constantly seeking sustainable, low cost, energy efficient, and heterogeneously connected wireless solutions, which the current technologies are unable to offer. For instance, a simple WiFi network relying on the IEEE 802.11 standard ensures a reliable communication in indoor environments, but is unable to provide localization or tracking of products.

This work presents a polarimetric analysis of the wireless channel characteristics of a large industrial warehouse, with an emphasis on the multipath properties of the channel. Recently, European COST actions such as COST 2100 [3] and COST IC1004 [4] have promoted the inclusion of Dense Multipath Components (DMC) in radio channel models, after following the observation that specular paths alone are often insufficient to account for all multipath energy in a radio channel. [5]-[7] discuss the multipath properties of indoor radio channels. [8] discusses the polarization properties of an indoor office environment, whilst [9], [10] both concern a polarimetric analysis of the multipath properties of an industrial hall containing a great deal of metallic equipment, which distinguishes this environment from many other indoor environments.

Based on the results of a large measurement campaign in an industrial warehouse with over 1507 distinct transmitterreceiver positions, we analyze polarimetric results such as cross-polarization discriminations and DMC characteristics as a function of transmitter-receiver distance. In this way, the properties and distributions of these parameters can be analyzed statistically.

The structure of this paper is as follows. Section II describes the measurement campaign, whilst Section III focuses on the applied channel model and its multipath properties. Section IV presents the measurement results, and Section V summarizes this paper with a conclusion and ideas for future work.

\section{MEASUREMENTS}

\section{A. Measurement environment}

The indoor propagation measurements were carried out in a flower auction warehouse, namely Royal FloraHolland in the Netherlands. Fig. 1 shows the measurement area, which has a width of $50 \mathrm{~m}$ and a length of $50 \mathrm{~m}$. The ground floor was made out of concrete, whilst the ceiling was made out of a metallic structure with corrugated triangles in shape, of which its height varied between $8 \mathrm{~m}$ and $10 \mathrm{~m}$. On the sides of the measurement area, there were three passages at a height of $5.6 \mathrm{~m}$ connecting different sections of the warehouse (such as distribution zones, buffer zones for the flowers, etc). The walls along the corridors were made out of concrete or glass. In addition, several metallic tracks were laid out on the ground floor for trolleys to be moved on, carrying the flowers. All 
measurements were carried out after the auction hours when the measurement environment was empty, and no trolleys or people were present. In total, seven long trajectories were measured (denoted as track A, B ...G), as indicated in Fig. 1. We have measured a total number of 1507 different Tx-Rx positions along these tracks, of which the distances between Tx (fixed) and Rx (moved along these tracks) varied between $10 \mathrm{~m}$ and $47 \mathrm{~m}$.

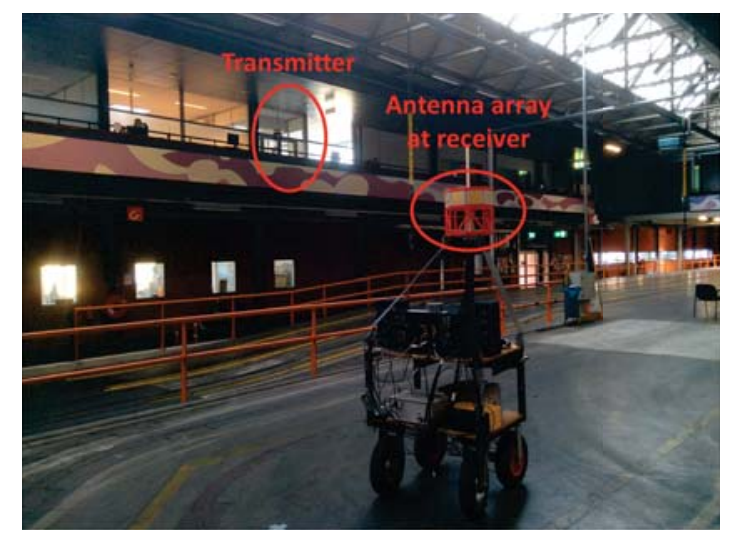

(a) View of transmitter and tracks A and B.

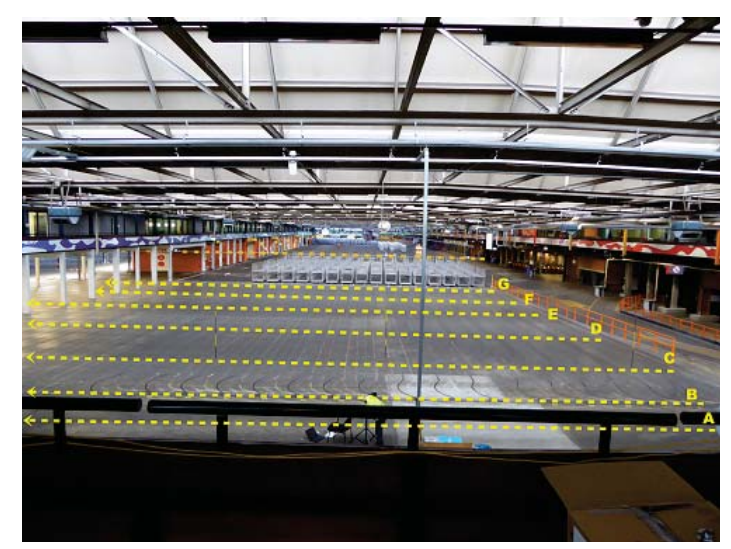

(b) View from transmitter to tracks A, B, C, D, E, F and G.

Fig. 1: Measurement environment

\section{B. Measurement scenario}

Multidimensional radio channel sounding measurements were performed using a dedicated Multiple-Input MultipleOutput (MIMO) channel sounder MIMOSA, jointly developed by IEMN/TELICE, University of Lille 1 (France) and INTECWAVES, Ghent University / imec (Belgium) [11]. MIMOSA enables real-time parallel channel sounding, where the data from each transmitting antenna is modulated simultaneously onto the carriers employing Interleaved Frequency Division Multiple Access (IFDMA). This enables us to measure the radio channel with an acquisition time of $350 \mu \mathrm{s}$. The aim of this measurement campaign was to characterize the multipath properties of the industrial radio channel in a large warehouse environment. We have sampled the radio channel transfer function in the frequency, space and polarization domains at a center frequency of $1.35 \mathrm{GHz}$, with a total number of 819 subcarriers occupying an $80 \mathrm{MHz}$ bandwidth.

A circular antenna arrays consisting of 8 dual-polarized patch antennas was used both at the transmitter (Tx) and the receiver $(\mathrm{Rx})$, in an $45^{\circ}$-slanted orientation with respect to the $\mathrm{x}$-axis. This allows us to measure both the diagonal $\left(+45^{\circ}\right)$ and anti-diagonal $\left(-45^{\circ}\right)$ radio channels, from which we can calculate the horizontally- $(\mathrm{H})$ and vertically $(\mathrm{V})$ polarized radio channels between $\mathrm{Tx}$ and $\mathrm{Rx}$ by applying Jones calculus. By doing so, we can measure the $16 \times 16$ full polarimetric (HH, HV, VH and VV) radio channel between Tx and Rx. As can be seen from Fig. 1a, the Tx antenna array was placed on the first floor, mounted at a height of $6 \mathrm{~m}$, whilst the $\mathrm{Rx}$ antenna array was placed on the ground floor, mounted at a height of $2 \mathrm{~m}$. The transmit power was $14 \mathrm{dBm}$.

\section{ChanNEL MODEL}

\section{A. Specular-and dense multipath components}

The radio channel can be seen as a combination of Specular Multipath Components (SMCs), Dense Multipath Components (DMC), and measurement noise. The SMCs represent the deterministic part of the radio channel, consisting of a number of plane waves with well defined parameters in the spatial, frequency, and time-delay domain. The DMC represent the stochastic part of the radio channel, which are continuous in the aforementioned dimensions, and originate mainly from diffuse scattering on electrically small and rough surfaces.

The measured radio channel can be expressed with the complex gain vectors $\boldsymbol{h}_{X Y} \in \mathbb{C}^{M_{R} M_{T} M_{f} \times 1}$, in which $M_{R}$ is the number of Rx antennas, $M_{T}$ the number of Tx antennas, and $M_{f}$ the number of frequency subcarriers. The complex gain $\boldsymbol{h}_{X Y}$ can be denoted as a summation over the SMC $s_{X Y}$, the DMC $\boldsymbol{d}_{X Y}$, and the noise $\boldsymbol{n}_{X Y}$ as follows:

$$
\boldsymbol{h}_{X Y}=\boldsymbol{s}_{X Y}\left(\boldsymbol{\theta}_{s, X Y}\right)+\boldsymbol{d}_{X Y}\left(\boldsymbol{\theta}_{d, X Y}\right)+\boldsymbol{n}_{X Y}\left(\sigma_{X Y}^{2}\right) \text {. }
$$

The subscripts $X$ and $Y$ in Eq. (1) denote the polarization of Tx and Rx, respectively. Both $X$ and $Y$ are either $\mathrm{H}$ - or Vpolarized. The vector $\boldsymbol{h}_{X Y}$ can be seen as a random variable, following a Gaussian distribution as follows [12]:

$$
\boldsymbol{h}_{X Y} \sim \mathcal{N}_{c}\left(\boldsymbol{s}_{X Y}\left(\boldsymbol{\theta}_{s, X Y}\right), \boldsymbol{R}_{X Y}\left(\boldsymbol{\theta}_{d, X Y}, \sigma_{X Y}^{2}\right)\right) .
$$

Both the DMC $\boldsymbol{d}_{X Y}$ as well as the measurement noise $\boldsymbol{n}_{X Y}$ can be characterized by the covariance matrix $\boldsymbol{R}_{X Y}$ in (2), which has the following structure [12]:

$$
\begin{aligned}
& \boldsymbol{R}_{X Y}\left(\boldsymbol{\theta}_{d, X Y}, \sigma_{X Y}^{2}\right) \\
& =\boldsymbol{I}_{M_{R}} \otimes \boldsymbol{I}_{M_{T}} \otimes \boldsymbol{R}_{f, X Y}\left(\boldsymbol{\theta}_{d, X Y}\right)+\sigma_{X Y}^{2} \boldsymbol{I}_{M} .
\end{aligned}
$$

In (3), $\boldsymbol{I}_{n}$ denotes an identity matrix of size $n$, and $\otimes$ represents the Kronecker product. The parameter $M$ denotes the total number of observations, such that $M=M_{R} M_{T} M_{f}$. In (3), we assume the measurement noise to be independent and identically distributed (i.i.d.) complex Gaussian noise with variance $\sigma_{X Y}^{2}$. In this work, we assume that the DMC covariance matrix $\boldsymbol{R}_{d, X Y}$ is uncorrelated in the spatial domains at 
both Tx and Rx. This implies that the DMC have a uniform power in the spatial domain (also called the angular domain), such that they can be represented with an identity matrix. It should be noted that works such as [13] or [14] propose to model the DMC in the spatial domain as clusters around the SMCs. We further assume $\boldsymbol{R}_{d, X Y}$ to be correlated in the frequency domain $\left(\boldsymbol{R}_{f, X Y}\right)$ [12], after observing that the Power Delay Profile (PDP) of the DMC $\psi_{X Y}(\tau)$ follows an exponential decaying function with time-delay $\tau$ :

$$
\psi_{X Y}(\tau)=\alpha_{d, X Y} e^{-\left(\frac{\tau-\tau_{d, X Y}}{\tau_{r e v}, X Y}\right)} .
$$

In (4), the parameters $\alpha_{d, X Y}, \tau_{r e v, X Y}$, and $\tau_{d, X Y}$ describe the polarization properties of the DMC in each sub-channel $X Y$, which are collected in the DMC parameter vector $\boldsymbol{\theta}_{d, X Y}$.

\section{B. RiMAX multipath estimator}

We have used the RiMAX multipath estimation framework detailed in [12] to obtain the maximum-likelihood estimates of $\hat{\boldsymbol{\theta}}_{s, X Y}, \hat{\boldsymbol{\theta}}_{d, X Y}$, and $\hat{\sigma}_{X Y}^{2}$ from the multidimensional channel sounding measurements. In the implementation of the RiMAX framework, we have accounted for the Effective Aperture Distribution Function (EADF) framework detailed in [15], which compensates for the influence of the $\mathrm{Tx}$ and $\mathrm{Rx}$ radiation patterns during the estimation of the multipath parameters.

We refer to [12] for a detailed description about the RiMAX framework and its implementation, as well as for a comprehensive description of the SMC parameter vector and the calculation of the SMC power spectrum.

\section{RESUlts}

\section{A. Estimated SMCs and DMC power spectra}

Fig. 2 shows an example of a measured PDP, together with the RiMAX-estimated SMCs and the DMC power spectrum.

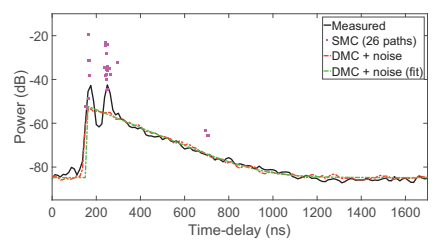

(a) HH-polarization

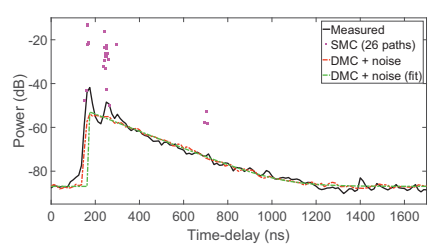

(c) VH-polarization

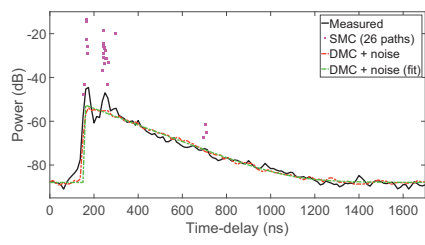

(b) HV-polarization

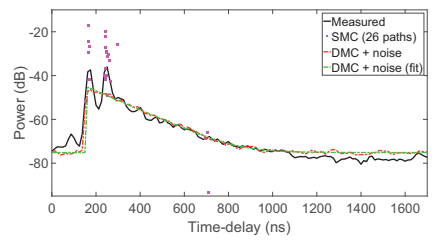

(d) VV-polarization
Fig. 2: Power Delay Profiles (PDPs) of the different polarizations for the measured channel, as well as the estimated SMCs and the DMC power spectra for track D, measurement position 1 (most right position of track D in Fig. 1b). The corresponding Tx-Rx distance was $26.4 \mathrm{~m}$.

From Fig. 2, we can observe that all relevant propagation paths (SMCs) are very well detected from the measured PDP by the RiMAX algorithm. We can also observe that the remaining DMC spectrum has an exponential power decay over time-delay, as we assumed in Eq. (4). In Fig. 2, this corresponds with a linear power decay in a dB-scale. We have also analyzed how much power in the measured radio channel was attributable to which polarization, of which the results in percentage can be found in Table I. No significant impact of the Tx-Rx distance on the relative polarimetric power was found (Pearson correlation coefficients ranging between -0.14 and -0.05 for the different polarizations).

\begin{tabular}{|c|c|c|c|c|c|}
\hline \multicolumn{2}{|c|}{} & HH & HV & VH & VV \\
\hline $\mathbf{P}_{\mathbf{X Y}}$ & $\mu$ & 0.17 & 0.11 & 0.12 & 0.61 \\
$\sum_{\mathbf{X}, \mathbf{Y} \in\{\mathbf{H}, \mathbf{V}\}} \mathbf{P}_{\mathbf{X Y}}$ & $\sigma$ & 0.03 & 0.03 & 0.03 & 0.06 \\
\hline
\end{tabular}

TABLE I: Relative power of each polarization sub-channel $(\mathrm{HH}, \mathrm{HV}, \mathrm{VH}$ and $\mathrm{VV}$ ) present in the measured power. $\mu$ represents the mean values, $\sigma$ represents the standard deviation. Results obtained from 1507 Tx-Rx measurements.

From Table I, we can state that the VV-polarization accounts for up to $60 \%$ of the measured power, and that both copolarizations ( $\mathrm{HH}$ and $\mathrm{VV}$ ) represent $80 \%$ of the measured power, whilst the cross-polarizations ( $\mathrm{HV}$ and $\mathrm{VH}$ ) only represent $20 \%$ of the measured power.

\section{B. Cross-polarization discrimination}

In this work, we analyze among others the cross-polar discrimination (XPD). The XPD is a qualitative metric to characterize the amount of de-polarization that has occurred in the radio channel between Tx and Rx. More precisely, it defines the proportion of the transmitted power of a polarization $(\mathrm{H}$ or $\mathrm{V})$ that is maintained in that polarization at the receiver end. Due to propagation mechanisms such as scattering and diffraction in the radio channel, ellipticity will occur to the polarization of the transmitted signal, resulting in so-called de-polarization. At the receiver, the incident signal will be a mixture of the transmitted polarized waves (e.g., $\mathrm{H}$ ), and de-polarized waves (in this case, V), resulting in a mixture of both $\mathrm{H}$ - and $\mathrm{V}$-polarized waves. The cross-polar discrimination is the ratio between the power maintained in the transmitted polarization (total co-polarized received power) to the power leaked into the other polarization (total crosspolarized received power). Eq. (5) and Eq. (6) denote the XPD for the $\mathrm{H}$ - and $\mathrm{V}$-polarizations, respectively:

$$
\begin{aligned}
& \left.X P D_{H}\right|_{\mathrm{dB}}=10 \log _{10}\left(\frac{\sum_{\tau} P_{H H}(\tau)}{\sum_{\tau} P_{H V}(\tau)}\right), \\
& \left.X P D_{V}\right|_{\mathrm{dB}}=10 \log _{10}\left(\frac{\sum_{\tau} P_{V V}(\tau)}{\sum_{\tau} P_{V H}(\tau)}\right) .
\end{aligned}
$$

The characterization of the amount of de-polarization is a very important metric to take into account in the design of wireless communication systems, since de-polarization can significantly degrade its performance, resulting in lower data rates and wasted radio spectrum. Higher XPD values denote less de-polarization, indicating that a large portion of the transmitted polarization is maintained in that polarization at the receiver, which is thus evidently preferred in communication 
systems. Fig. 3 shows the cumulative distribution function (CDF) of the XPD values for the H- and V-polarization of the measured radio channel, as well as of the SMC and the DMC power spectra. No significant impact of the Tx-Rx distance on the XPD values was found (Pearson correlation coefficients ranged between -0.14 and -0.01 for the different polarizations).

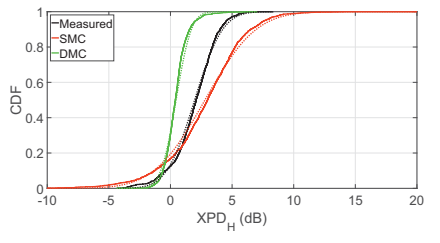

(a) CDF of $X P D_{H}$-values

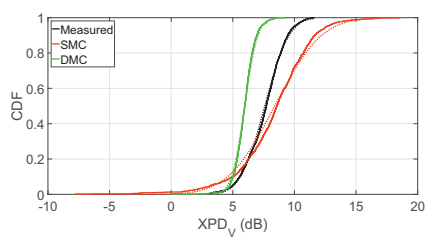

(b) $\mathrm{CDF}$ of $X P D_{V}$-values
Fig. 3: $C D F$ of the XPD values (in $\mathrm{dB}$ ) of the measured channel, as well as of the SMC and the DMC power spectra of the radio channel. Full lines indicate measured values, dotted lines represent their fit with a normal distribution.

Fig. 3 shows that we can model the XPD values (in $\mathrm{dB}$ ) of the measured channel and the SMC and the DMC parts of the radio channel fairly well with a normal distribution (such that their non-logarithmized values are log-normally distributed).

Table II summarizes the main results of this analysis, in which $p_{10}, p_{50}$ and $p_{90}$ denote the 10 -th percentile, the 50th percentile (median), and the 90-th percentile of the XPD values, respectively.

\begin{tabular}{|c|c|cc|cc|cc|}
\hline \multicolumn{2}{|c|}{} & \multicolumn{2}{|c|}{ Measured } & \multicolumn{2}{c|}{ SMC } & \multicolumn{2}{c|}{ DMC } \\
\cline { 3 - 8 } \multicolumn{2}{|c|}{} & $\mathrm{H}$ & $\mathrm{V}$ & $\mathrm{H}$ & $\mathrm{V}$ & $\mathrm{H}$ & $\mathrm{V}$ \\
\hline \multirow{3}{*}{ XPD (dB) } & $p_{10}$ & -0.41 & 5.55 & -1.27 & 4.98 & -0.61 & 5.03 \\
& $p_{50}$ & 2.08 & 7.72 & 3.01 & 8.57 & 0.40 & 5.97 \\
& $p_{90}$ & 4.03 & 9.45 & 6.50 & 11.65 & 1.55 & 6.95 \\
\hline
\end{tabular}

TABLE II: Cross-polarization discrimination (XPD) values (in $\mathrm{dB}$ ) of the measured channel, as well as of the SMC and the DMC power spectra of the radio channel. $\mathrm{H}$ denotes the $X P D_{H}$ value, whilst $\mathrm{V}$ denotes the $X P D_{V}$ value. Results obtained from 1507 Tx-Rx measurements.

From Fig. 3 and Table II, we can observe that the Vpolarized transmitted waves maintain up to 5 to $6 \mathrm{~dB}$ more of their power when impinging at the receiver, compared to the H-polarized transmitted waves. This means that the radio channel will depolarize the H-polarized waves more than the V-polarized waves. Furthermore, the $X P D_{H}$ values of the DMC part of the radio channel are close to zero, indicating that diffuse reflections will cause the $\mathrm{H}$-polarized transmitted waves to get de-polarized nearly entirely.

\section{DMC power ratio}

One of the aspects that we wanted to quantify in this study was the relative power attributable to the DMC part of the measured radio channel in the industrial warehouse. We can write this DMC power ratio as follows for each combination of polarizations $X Y$ :

$$
p_{D M C, X Y}=\frac{\sum_{\tau} P_{D M C, X Y}(\tau)}{\sum_{\tau} P_{(S M C+D M C), X Y}(\tau)} .
$$

We have found a weak correlation between Tx-Rx distance and DMC power ratio, of which the Pearson correlation coefficient was about 0.15 for the cross-polarizations, and 0.20 for the copolarizations. Similar conclusions were found in [10], which also reports a strong dependence of the DMC power ratio with polarization, but not with Tx-Rx distance. Fig. 4 shows the CDF of the DMC power ratio for each combination of polarizations $X Y$ :

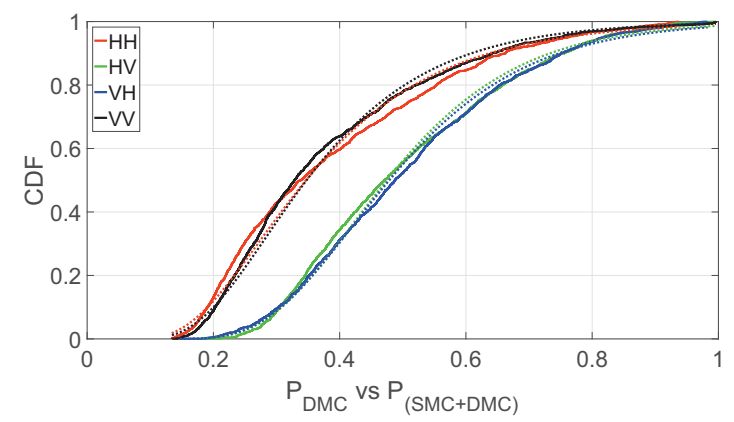

Fig. 4: $\mathrm{CDF}$ of the DMC power ratio as a function of Tx-Rx distance for $\mathrm{HH}, \mathrm{HV}, \mathrm{VH}$ and VV polarizations. Full lines indicate measured values, dotted lines represent their fit with an inverse Gaussian distribution.

A Wilcoxon rank-sum test confirmed that there is a group effect on both the cross-polarized DMC power ratios at the $5 \%$ significance level, and also on both the co-polarized DMC power ratios (p-values of 0.95 and 0.19 , respectively). However, a Kruskal-Wallis test confirmed that cross- and copolarized DMC power ratios come from a different distribution ( $\mathrm{p}$-value of less than $1 \times 10^{-169}$ ), which is also apparent from Fig. 4. This observation was also reported in [10].

Table III summarizes the results of this analysis, in which $p_{10}, p_{50}$ and $p_{90}$ denote the 10 -th percentile, the 50-th percentile (median), and the 90-th percentile of the DMC power ratios, respectively.

\begin{tabular}{|c|c|c|c|c|c|}
\hline \multicolumn{2}{|l|}{} & HH & HV & VH & VV \\
\hline $\mathbf{P}_{\text {DMC }}$ & $p_{10}$ & 0.18 & 0.30 & 0.29 & 0.19 \\
$\mathbf{P}_{\text {(SMC+DMC })}$ & $p_{50}$ & 0.33 & 0.47 & 0.48 & 0.32 \\
& $p_{90}$ & 0.65 & 0.78 & 0.80 & 0.59 \\
\hline
\end{tabular}

TABLE III: DMC power ratio of the full polarimetric channel $(\mathrm{HH}, \mathrm{HV}, \mathrm{VH}$ and VV). Results obtained from 1507 Tx-Rx measurements.

From Table III, we can conclude that there is about 10 to $20 \%$ more power in the DMC part of the cross-polarizations than in those of the co-polarizations. This can be explained by the fact that the power in the cross-polarizations is dominated by scattered paths, which suffer heavily from de-polarization. Meanwhile, the power in the co-polarizations is mainly dominated by the direct path between $\mathrm{Tx}$ and $\mathrm{Rx}$, which does not undergo de-polarization since $\mathrm{Tx}$ and $\mathrm{Rx}$ are in line-of-sight.

The DMC power ratios ranged between about $15 \%$ and $80 \%$, which is higher than the reported values of $23-38 \%$ in [9] at $3 \mathrm{GHz}$ in an industrial environment. This can be explained by the fact that there is inherently more diffuse scattering at $1.35 \mathrm{GHz}$ than for higher frequencies [8], since the 
surfaces on which the propagation paths reflect are electrically smaller, and are therefore perceived as more rough, resulting in more diffuse scattering.

\section{DMC reverberation time}

In this subsection, we look into the behavior of the DMC reverberation time, denoted by the variable $\tau_{d, X Y}$ in Eq. (4), which is the time for the electromagnetic waves to uniformly distribute themselves across a room. According to the theory of Room Electromagnetics (RE) [16], it only depends on the volume of the room, its surface area and an effective absorption coefficient, and is assumed to be constant across the entire room. In Eq. (4), $\tau_{d, X Y}$ regulates the slope of the exponential decay in the PDP of the DMC, which corresponds with a linear decay in a dB-scale. Fig. 5 shows the DMC reverberation time as a function of Tx-Rx distance for each combination of polarizations $X Y$ :

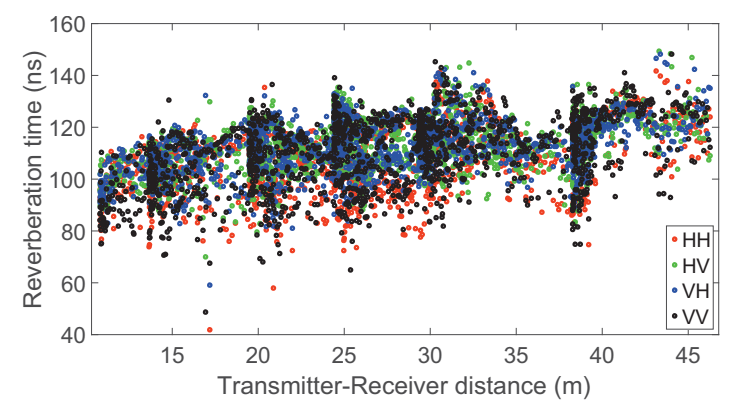

Fig. 5: DMC reverberation time as a function of Tx-Rx distance for $\mathrm{HH}, \mathrm{HV}$, $\mathrm{VH}$ and VV polarizations.

Fig. 5 shows that Tx-Rx distance and reverberation time are correlated with each other, of which the values for the latter ranged from $80 \mathrm{~ns}$ for distances around $10 \mathrm{~m}$, up to $130 \mathrm{~ns}$ for distances up to $45 \mathrm{~m}$. This correlation was supported by analyzing the Pearson correlation coefficients between Tx-Rx distance and reverberation time, which were 0.41 for $\mathrm{HH}, 0.32$ for $\mathrm{HV}, 0.44$ for $\mathrm{VH}$ (p-values less than $\left.1 \times 10^{-35}\right)$. However, for the VV-polarization, this coefficient was only 0.05 with a p-value of 0.06 . This implies that only the VV-polarization is subject to the theory of RE. A Wilcoxon rank-sum test confirmed that there is a group effect on both the cross-polarized DMC reverberation times at the $5 \%$ significance level, but rejects this group effect on both the co-polarized DMC reverberation times ( $\mathrm{p}$-values of 0.45 and 0.0006 , respectively).

\section{CONCLUSIONS}

This work presented a polarimetric analysis of the specularand dense multipath components (SMCs and DMC) in a large industrial warehouse, based on a MIMO channel sounding measurement campaign with 1507 distinct positions of transmitter and receiver. Based on the results of a cross-polar discrimination analysis, and looking into the relative power ratio of the $\mathrm{DMC}$, we highlight the fact that $\mathrm{DMC}$ has a significant effect on the total power in the radio channel in industrial warehouses, representing up to $80 \%$ of the measured power. We have also found a clear distinction between $\mathrm{H}$ - and V-polarized waves, both on their tendency to de-polarize, as well as their influence on the total power in the radio channel.

\section{ACKNOWLEDGMENT}

Brecht Hanssens is funded by a Ph.D. grant of the Agency for Innovation by Science and Technology (IWT). Emmeric Tanghe is a Post-Doctoral Fellow of the Research Foundation Flanders (FWO). This research is part of the HYCOWARE project realized in collaboration with IMEC. Project partners are Egemin, Aucxis and Intation, with project support from VLAIO (Flanders Innovation and Entrepreneurship).

The authors would like to thank Matthias Van den Bossche, M. Eng., and Rose Mazari, M. Eng., for their support during the measurement campaign.

\section{REFERENCES}

[1] LoRA Alliance. LoRAWAN 1.0.2 Specification. LoRA Document, 2016

[2] ZigBee Alliance. ZigBee Specification v1.0. ZigBee Document 053 $473 r 00,2005$.

[3] R. Verdone and A. Zanella. Radio Channel Modeling for 4G Networks. In Pervasive Mobile and Ambient Wireless Communications: COST Action 2100, Signals and Communication Technology, pages 67-148. Springer, 2012.

[4] N. Cardona. Cooperative Radio Communications for Green Smart Environments: COST IC1004. The River Publishers Series in Communications. River Publishers, 2016.

[5] A. Richter, J. Salmi, and V. Koivunen. Distributed Scattering in Radio Channels and its Contribution to MIMO Channel Capacity. In European Conference on Antennas and Propagation (EuCAP), pages 1-7, 2006.

[6] M Kaske, M. Landmann, and R. Thomä. Modelling and Synthesis of Dense Multipath Propagation Components in the Angular Domain. European Conference on Antennas and Propagation (EuCAP), (7):26412645, 2009.

[7] J. Poutanen, J. Salmi, K. Haneda, et al. Angular and Shadowing Characteristics of Dense Multipath Components in Indoor Radio Channels. IEEE Transactions on Antennas and Propagation, 59(1):1-9, 2011.

[8] B. Hanssens, M.-T. Martinez-Ingles, E. Tanghe, et al. Polarimetric Analysis of Reverberation Times for $94 \mathrm{GHz}$ Indoor Communication. In European Conference on Antennas and Propagation (EuCAP), pages 700-702, 2017.

[9] E. Tanghe, D. P. Gaillot, M. Liénard, et al. Experimental Analysis of Dense Multipath Components in an Industrial Environment. IEEE Transactions on Antennas and Propagation, 62(7):3797-3805, 2014.

[10] D. P. Gaillot, E. Tanghe, W. Joseph, et al. Polarization Properties of Specular and Dense Multipath Components in a Large Industrial Hall. IEEE Transactions on Antennas and Propagation, 63(7):3219-3228, 2015.

[11] P. Laly, D. P. Gaillot, M. Liénard, et al. Flexible real-time channel sounder for multidimensional polarimetric parameter estimation. IEEE Conference on Antenna Measurement and Application, 1, 2015.

[12] A. Richter. Estimation of Radio Channel Parameters: Models and Algorithms. $\mathrm{PhD}$ thesis, Technische Universität Ilmenau, Fakültat für Elektrotechnik und Informationstechnik, Ilmenau, DE, 2005.

[13] J. Poutanen, J. Salmi, K. Haneda, et al. Propagation Characteristics of Dense Multipath Components. IEEE Antennas and Wireless Propagation Letters, 9:791-794, 2010.

[14] F. Quitin, C. Oestges, F. Horlin, et al. Diffuse Multipath Component Characterization for Indoor MIMO Channels. In European Conference on Antennas and Propagation (EuCAP), pages 1-5, 2010.

[15] M. Landmann. Limitations of Experimental Channel Characterisation. $\mathrm{PhD}$ thesis, Technische Universität Ilmenau, Fakültat für Elektrotechnik und Informationstechnik, Ilmenau, Germany, 2008.

[16] J. B. Andersen, J. O. Nielsen, G. F. Pedersen, et al. Room Electromagnetics. IEEE Antennas and Propagation Magazine, 49(2):27-33, 2007. 